\title{
Treatment of chronic active hepatitis in children
}

\author{
Reuben S. Dubois \\ M.B., B.S. \\ Arnold Silverman \\ M.D. \\ Department of Pediatrics, University of Colorado Medical Center, Denver, Colorado, \\ and Children's Hospital, Denver
}

\begin{abstract}
Summary
Thirty-eight children with chronic active hepatitis are reported. Their mode of presentation was usually indistinguishable from acute viral hepatitis (63\%). Extra hepatic manifestations were more common in those patients with an insidious onset. The twentyeight patients tested for hepatitis B antigen (HB Ag) were all negative and SGOT values were higher (mean 1000 i.u./l) than those reported in adult patients with chronic active hepatitis. Thirty-three patients had marked elevation of serum gammaglobulin (mean $3.78 \mathrm{~g} / 100 \mathrm{ml}$ ) and $34 \%$ had positive LE cells. All patients had histologic features of 'aggressive' hepatitis; in addition five biopsies also showed areas of submassive hepatic necrosis. The initial response to corticosteroid was excellent in all but three patients. To date drug therapy has been suspended in fifteen patients for periods of 3 months to 4 years and 7 months. There have been eight deaths. Three with hepatic insufficiency and two with sepsis. Three others have subsequently died following orthotopic liver transplantation.
\end{abstract}

\section{Introduction}

Although the literature contains many reviews of chronic active hepatitis in adults, Mistilis and Blackburn (1970), Read, Sherlock and Harrison (1963), Soloway et al. (1972), Baggenstoss et al. (1972) there are few reports which deal exclusively with this entity in the paediatric age group and are based on a significant number of patients, Page, Good and Pollora (1969), Dubois, Silverman and Slovis (1972).

This report concerns our experience with thirtyeight children with chronic active hepatitis, the majority having presented in the last 5-7 years. Follow-up of these patients has provided an opportunity to determine the effect of corticosteroid and azathioprine therapy on the course of children with chronic active hepatitis. In addition, observation of these patients has revealed certain clinical and biochemical differences between paediatric and adult patients with this syndrome.

\section{Materials and methods} Clinical

The patient group was composed primarily of 0 referral patients drawn from a large geographic area of urban and rural composition. The diagnosis of $\stackrel{\circ}{\sigma}$ chronic active hepatitis was made on accepted 을 clinical, biochemical, immunologic and histologic criteria, Soloway et al. (1972), Baggenstoss et al. $\subsetneq$ (1972). Thirty-six of the patients represented sporadic $\overbrace{\overparen{D}}$ cases since neither contact with known jaundice subjects nor secondary spread could be establishe $\vec{G}$ In one patient there was definite contact with hepatitis A, while another developed chronic actie hepatitis 5 years after her sibling.

The mean age for this patient group was 10 years and 2 months (range, 3 years to 17 years and 6 months), thirty patients were female. The disease began acutely, with a prodrome of anorexia, nausea and fever in twenty-four patients. Jaundice was present in all patients with this type of onset. The remaining fourteen patients had a more insidious onset. All but five of this group were also jaundiced at the time of diagnosis. Twenty-three of the patients had persistent jaundice from the beginning of their illness. Additional clinical features are summarized in Table 1.

Ascites was present in nine patients before treat- 3 ment and developed rapidly in three others shortly 을 after corticosteroids were started. Seven patients had episodes of either hepatic coma or pre-coma during the course of their illness. Oesophageal varices were

TABle 1. Significant physical findings at diagnosis in thirty-eight children with chronic active hepatitis

\begin{tabular}{lr}
\hline Jaundice & 33 \\
Hepatomegaly & 30 \\
Splenomegaly & 28 \\
Ascites & 9 \\
Digital clubbing & 6 \\
Amenorrhea & 7 \\
Acne & 6 \\
Gynaecomastia & 2 \\
\hline
\end{tabular}


found in two patients at the time of diagnosis and developed in four additional cases later in the disease. Amenorrhea was present in seven of eight patients in whom menses had started prior to onset of disease. Acne was noted only in the adolescent age child, rendering the significance of this finding questionable. Likewise, the presence of gynaecomastia in the two teenage male patients may also be a normal variant. The presence of spider angiomata in this age group are difficult to evaluate unless they are on the chest or back.

TABLE 2. Extra hepatic manifestations occurring in fifteen of thirty-eight patients with chronic active hepatitis

\begin{tabular}{ll}
\hline Arthralgia, arthritis & 9 \\
Erythaema nodosum & 3 \\
Acute colitis & 3 \\
Thyroiditis & 2 \\
Diabetes & 2 \\
Haematuria & 3 \\
Parotitis & 1 \\
Pleurisy & 1 \\
Thrombophlebitis & 1 \\
\hline
\end{tabular}

Extra-hepatic manifestations which may have had an auto-immune basis occurred in fifteen patients, some demonstrating multiple system involvement (Table 2). Erythema nodosum occurred only in those patients who had arthritis. Colitis was diagnosed by proctosigmoidoscopy, rectal biopsy and barium enema in three patients. Diabetes occurred in two patients on corticosteroids, with thyroiditis as a subsequent manifestation in one patient. Haematuria was present in three patients and associated with low serum complement in the one patient tested. Another had proliferative glomerulonephritis on renal biopsy.

\section{Laboratory \\ Biochemical}

The majority of the patients had a large number of laboratory tests with serum total bilirubin, serum glutamic oxaloacetic transaminase, prothrombin time and serum protein electrophoresis evolving as the most useful. The results of these determinations obtained at the time of diagnosis are summarized in Fig. 1. Thirty-one patients had serum total bilirubin levels less than $10 \mathrm{mg} / 100 \mathrm{ml}$. SGOT values ranged from 65 to 3640 i.u./l, with a mean of 1000 . Serum albumin tended to be low or low normal in most patients (range 1.8 to $4.0 \mathrm{~g} / 100$ ) with a mean of 2.97 . The serum gammaglobulin ranged from 2.0 to 6.0 $\mathrm{g} / 100 \mathrm{ml}$ with a mean of 3.78 . Prothrombin time was seldom below $30 \%$ of normal, however, more than half (twenty patients) had a prothrombin time of less than $50 \%$.
Immunochemical

A variety of immunochemical studies were performed and the results are summarized in Table 3.

\section{Histologic}

Liver tissue was obtained during life, by either open (19) or closed (17) methods in thirty-six patients and at autopsy in two others.

TABLE 3. Immunochemical results at diagnosis in children with chronic active hepatitis

\begin{tabular}{llc}
\hline & \multicolumn{2}{c}{ No. of patients } \\
\cline { 2 - 3 } & Total & Positive \\
\hline Serum immunoglobulins & & \\
(Elevated IgG for age) & 14 & 14 \\
Anti-nuclear antibody & 23 & 14 \\
LE cells & 33 & 13 \\
Hepatitis B antigen & 28 & 0 \\
\hline
\end{tabular}

Histologic evidence for 'aggressive' hepatitis was present in all biopsies characterized by periportal extension of inflammatory response (plasma cells and lymphocytes), loss of the limiting plate and piecemeal necrosis. Six patients had the pattern of subacute hepatitis with multilobular necrosis (submassive hepatic necrosis) in addition to the other features. In the latter group the onset was abrupt or there was an acute exacerbation in a previously insidious course. Serum total bilirubin was usually greater than $20 \mathrm{mg} / 100 \mathrm{ml}$ and the prothrombin time was less than $30 \%$ of normal (see Fig. 1).

The histologic features of post-necrotic cirrhosis were already present at the time of diagnosis in sixteen patients. In view of the known patchy nature of the hepatic lesion in chronic active hepatitis and also the sampling error in closed needle biopsy of cirrhosis, Soloway et al. (1971), this number might actually have been higher had all patients been subjected to open biopsy.

\section{Results \\ Treatment}

All but one of our patients had symptoms which interfered with their normal life-styles. It is our feeling, however, that all children in whom the diagnosis of chronic active hepatitis is established, should be treated whether they are symptomatic or not. Thirty-five patients completely fulfilled the accepted criteria for diagnosis and thirty-four were started on prednisone $(2 \mathrm{mg} / \mathrm{kg} /$ day $)$ from 1 month to 3 years after the apparent onset of the disease (median 5.8 months). Three additional patients were started on therapy on the basis of only clinical, biochemical and immunochemical evidence, since liver biopsy was contraindicated. The diagnosis was 


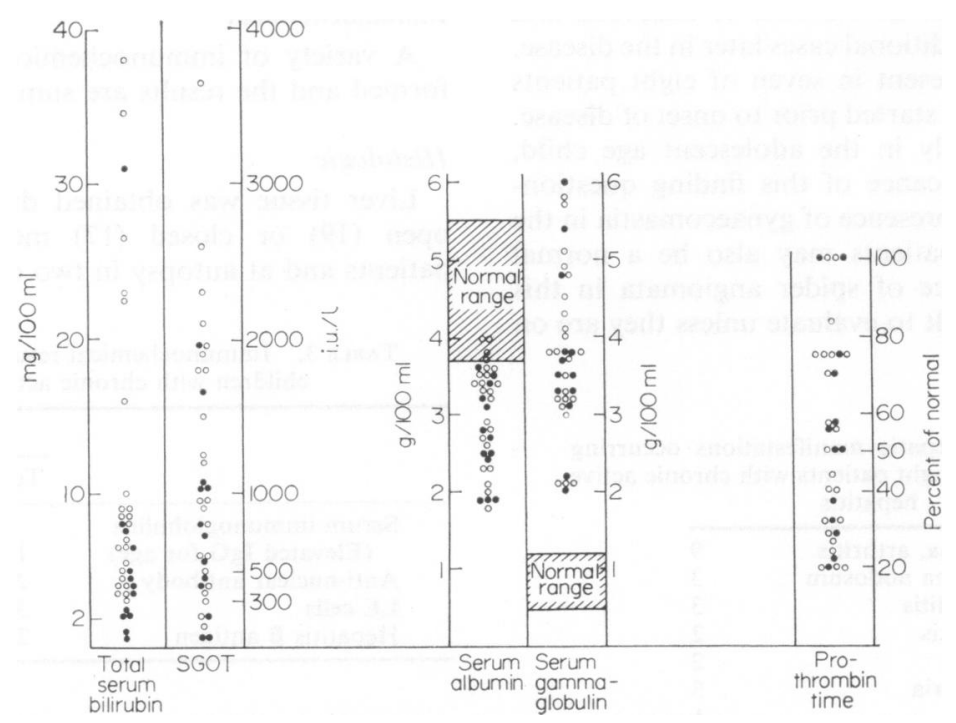

FIG. 1. Results of biochemical laboratory tests obtained at time of diagnosis in thirty-eight children with chronic active hepatitis. $O$, insidious onset; $O$, acute onset.

TABLE 4. Clinical course of thirty-eight children with chronic active hepatitis

\begin{tabular}{lc}
\hline \multicolumn{1}{c}{ Clinical course } & No. of patients \\
\hline Prompt clinical remission (on Rx) & 33 \\
Clinical and biochemical remission (on Rx) & 25 \\
Sustained remission (off Rx) & 15 \\
Deaths & 8 \\
\hline
\end{tabular}

subsequently confirmed histologically at autopsy in two of these subjects and in the third, 2-3 months after the institution of therapy at which time the bleeding diathesis had markedly improved.

Azathioprine $(1 \mathrm{mg} / \mathrm{kg} /$ day $)$ was added to the therapeutic regimen in twenty-two patients. However, this therapy was usually withheld until clinical and biochemical improvement appeared. Serial measurements of white blood cells and platelet counts were performed after the introduction of azathioprine. In all but three of the treated patients, attempts were then made to reduce the corticosteroid dosage to low maintenance levels $(10 \mathrm{mg}$ or less per day, or $10-20 \mathrm{mg}$ every other day) and were discontinued along with azathioprine in fifteen patients from 6 months to 4 years after initiating therapy. All fifteen had been in clinical and biochemical remission for periods of 5 months to 3 years. The majority, however, had been in remission from 18 to 24 months before slowly weaning from therapy. A low salt diet and diuretics were occasionally needed for management of ascites. Lasix ${ }^{\circledR} 0 \cdot 5-1$ $\mathrm{mg} / \mathrm{kg}$ given orally in two divided doses proved effective. Rarely was it necessary to add Aldactone- $A^{\circledR}$
$(1.5-3.0 \mathrm{mg} / \mathrm{kg} /$ day in four divided doses) to the $\vec{\varphi}$ therapeutic regimen. Azulfidine $(2-4 \mathrm{~g} /$ day $)$ wig $\$$ used with excellent symptomatic response in thre patients who developed acute colitis.

Orthotopic liver transplantation, Starzl (1969) was performed from 1 to 4 years after the onset, in five patients because of decompensated liver disease. Four had become refractory to medical therapy, while the fifth received no corticosteroid and/or azathioprine over the 4-year period of follow-up.

\section{Course}

Of the thirty-six symptomatic patients who were treated with prednisone thirty-three had a prompt clinical remission with disappearance of jaundice 3 . and improvement in well-being within 1-3 months $\delta$ of instituting therapy. In most cases this occurred prior to the addition of azathioprine to the thera- $\frac{\text { o }}{7}$ peutic regimen. A complete biochemical remission $\rightarrow$ occurred in twenty-five patients (fifteen combined, ten prednisone) with serum total bilirubin, SGOT and serum electrophoresis returning to normal. To date fifteen (eleven combined, four prednisone) of $N$ these twenty-five patients have been removed from $N_{\omega}^{N}$ drug therapy and have remained in clinical and bio- 0 chemical remission for periods of 3 months to 40 years and 7 months. Five of these patients had repeat $\frac{}{\Phi}$ closed liver biopsies. Two revealed a normal $\stackrel{\text { ? }}{?}$ histologic picture while the three others yielded $\frac{T}{0}$ inadequate tissue.

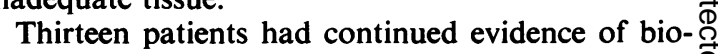
chemical activity with intermittent clinical relapse $\stackrel{\mathbb{D}}{\varrho}$ 
despite therapy in twelve (seven combined, five prednisone). In the majority, this activity was associated with mild increases in serum bilirubin with SGOT remaining two to five times normal (60-150 i.u./l) and with serum gammaglobulin more than twice normal.

Five of these thirteen patients had persistent marked hyperbilirubinaemia (serum total bilirubin greater than $20 \mathrm{mg} / 100 \mathrm{ml}$ ) and prothrombin time below $30 \%$ of normal, despite corticosteroid therapy. This finding was associated with a poor prognosis; three patients died, another lapsed into hepatic coma but survived while the fifth underwent orthotopic liver transplantation 1 year after the apparent onset of disease.

One other patient had marked hyperbilirubinaemia and prothrombin time below $30 \%$ of normal. This subject, however, improved markedly with corticosteroid therapy and presently is in clinical and biochemical remission 12 months after discontinuing therapy.

Repeat liver biopsies have been performed in six patients (five open and one autopsy) with persistent or ${ }^{x}$ recurrent clinical and biochemical abnormalities. All revealed evidence of cirrhosis with varying degrees of activity. Closed liver biopsies were performed on two patients in clinical and biochemical remission but still on therapy. In both cases inadequate tissue was obtained.

There have been eight deaths attributable to either the primary disease or the combination of disease and drug therapy. One patient died of hepatic insufficiency 1 week after prednisone was instituted. Another died of hepatic insufficiency within 6 months of the onset of disease, despite treatment with corticosteroids and azathioprine for $\mathbf{4}$ months. In another gastrointestinal bleeding, hepatic coma and death occurred 4-5 years after the institution of corticosteroid therapy. Two other patients died of sepsis while on both prednisone and azathioprine, one having pseudomonas sepsis in association with extensive subacute hepatitis with multilobular necrosis (submassive hepatic necrosis) the other shigellosis. Three other patients have subsequently died following orthotopic liver transplantation (Starzl, personal communication).

\section{Discussion}

There are many similarities between paediatric and adult patients with chronic active hepatitis, however, certain differences in clinical and biochemical patterns and therapeutic responses are worth noting.

In adults the onset is usually insidious in nature, often with extra hepatic manifestations predominating. In one large series (Mistilis and Blackburn 1970), only about one-third of patients had an abrupt onset with clinical and laboratory features indistinguishable from acture viral hepatitis. Conversely, the onset was acute in $63 \%$ of our patients. Page et al. (1969) also noted an abrupt onset in about $50 \%$ of their paediatric patients with chronic liver disease and hypergammaglobulinaemia. It is interesting to note that one-half of the corticosteroid treated group in the report by Cook, Mulligan and Sherlock (1971) also had an acute onset of jaundice. Furthermore, even though selection was randomized this group comprised younger patients when compared with the control group. Five of seven patients with chronic active hepatitis and $\mathrm{HB} \mathrm{Ag}$ reported by Bulkley et al. (1970) had an abrupt onset.

Conversely, although the onset was acute in the majority of our patients, $\mathrm{HB} \mathrm{Ag}$ was negative in all twenty-eight patients tested.

In adult patients SGOT rarely exceeds 500 i.u./1 and is a useful point when differentiating acute viral hepatitis from chronic active hepatitis, Mistilis and Blackburn (1970). In the present series the mean SGOT was significantly greater than this level. The reason for this difference is not known, however, this may be related to the frequent acute onset noted in younger patients. Although in the present series there was no correlation between the degree of SGOT elevation at the time of diagnosis and type of onset. Unfortunately, transaminase levels were not reported in the only other large series of children with chronic active hepatitis, Page et al. (1969).

Hypergammaglobulinaemia was present in every case, but not to the same degree observed in the children reported by Page et al. (1969). In contrast to the lack of diagnostic value of hypergammaglobulinaemia in adults, Mistilis and Blackburn (1970), it proved extremely useful in our experience in distinguishing chronic active hepatitis from either recurrent or persistent viral hepatitis.

The importance of excluding Wilson's disease in any young patient with hepatic dysfunction has been frequently stressed, Slovis et al. (1971), however, with appropriate studies Wilson's disease can be readily distinguished from chronic active hepatitis, Sternlieb and Scheinberg (1972). Similarly, druginduced hepatitis should always be considered, Goldstein, Lam and Mistilis (1973). In retrospect, none of our patients had taken oxyphenisatin or alpha methyl-dopa.

It is now well established that corticosteroid therapy significantly reduces the mortality in the early active phase of chronic active hepatitis, Mistilis and Blackburn (1970), Soloway et al. (1972), Cook et al. (1971), Murray-Lyon, Stern and Williams (1973). The majority of our patients had a prompt clinical remission with improved well-being following the institution of corticosteroid therapy. All three of our patients who died early in the course of the 
disease despite corticosteroid therapy had submassive hepatic necrosis.

The side effects of prolonged corticosteroid therapy (cosmetic, growth retardation, osteoporosis and increased protein catabolism) are usually more serious in children than adults. These side effects may occur even in the smaller corticosteroid dosage employed by Cook et al. (1971).

Combined azathioprine and prednisone is no more effective than prednisone alone in improving the various indicators of disease activity, Soloway et al. (1972). However, the introduction of azathioprine in the long-term management of chronic active hepatitis in children has been extremely beneficial in reducing untoward side-effects of corticosteroid therapy. Most of our patients could be maintained in clinical and biochemical remission while taking small doses of prednisone in combination with azathioprine.

Institution of azathioprine may lead to haematologic or hepatic complications, however, this was not a problem in our experience. Similarly, the risk of infection is enhanced with azathioprine therapy and in the present study two patients died with sepsis.

The effect of therapy on the biochemical abnormalities and the occurrence of relapse following discontinuation of therapy is variable, Read et al. (1963), Soloway et al. (1972), Page et al. (1969), Cook et al. (1971), Murray-Lyon, Stern and Williams (1973), Mackay (1968). Seventy-three per cent of our patients who received either long-term combination or prednisone therapy had a complete biochemical remission. This data is remarkably similar to the controlled study from the Mayo clinic, Soloway et al. (1972) but in contrast to a remission rate of about $20 \%$ reported by Cook et al. (1971). Whether the difference reflects the use of azathioprine in addition to low dose corticosteroid is not known.

The value of serial transaminase estimation in assessing or controlling drug therapy is controversial, Mistilis and Blackburn (1970), Cook et al. (1971). In our experience, slight increase in SGOT was not uncommon when attempts to reduce corticosteroid dosage were made early in the course of the disease. Untreated chronic active hepatitis not uncommonly 'burns out' after 2-3 years, and the transaminase may become normal, Cook et al. (1971). For this reason we have not discontinued therapy unless both the serum albumin and gammaglobulin in addition to SGOT have returned to normal levels.

Early clinical and biochemical relapse is common in chronic active hepatitis on cessation of therapy, Mackay (1968). In one large series this occurred in about $50 \%$ of cases, and usually within 6 months of discontinuation of therapy, Soloway et al. (1972). Fifteen of our patients who were in clinical and biochemical remission on treatment have had all therapy discontinued, and in contrast none has relapsed during the period of follow-up. The reason for this difference is not apparent at this time. A further period of observation and adequate liver biopsies will be necessary in order to determine whether these patients are 'cured'.

In contrast to the findings of Page et al. (1969) no clinical, biochemical, immunologic or histologic feature was of value in predicting the effect of treatment. Whether any of these indices or therapy modify the long-term prognosis awaits further observation.

\section{Acknowledgment}

Supported in part by a grant (RR-69) from the general clinical Research Centers Program of the Division of Research Resources, National Institutes of Health.

\section{References}

Baggenstoss, A.H., Soloway, R.D., Summerskill, W.H.J ., Elveback, L.R. \& SChOENField, L.J. (1972) Chronic active liver disease. The range of histologic lesions, their response to treatment and evaluation. Human Pathology, 3, 183.

Bulkley, B.H., Heizer, W.D., Goldfinger, S.E., IsSelBaCher, K.J. \& Shulman, N.R. (1970) Distinctions in chronic active hepatitis based on circulating hepatitisassociated antigen. Lancet, ii, 1323.

CoOk, G.C., Mulligan, R. \& Sherlock, S.O. (1971) Controlled prospective trial of corticosteroid therapy in active chronic hepatitis. Quarterly Journal of Medicine, 40, $15 \%$

Dubois, R.S., Silverman, A. \& Slovis, T.L. (1972) Chron还 active hepatitis in children. American Journal of Digestive Disease, 17, 575.

Goldstein, G.B., LaM, K.C. \& Mistilis, S.P. (1973) Drug induced active chronic hepatitis. American Journal of Digestive Disease, 18, 177.

MACKAY, I.R. (1968) Chronic hepatitis: effect of prolonged suppressive treatment and comparison of azathioprine with prednisolone. Quarterly Journal of Medicine, 37, 379.

Mistilis, S.P. \& Blackburn, C.R.B. (1970) Active chronic hepatitis. American Journal of Medicine, 48, 484.

Murray-Lyon, I.M., Stern, R.B. \& Williams, R. (1973) Controlled trial of prednisone and azathioprine in active chronic hepatitis. Lancet, i, 735.

Page, A.R., Good, R.A. \& Pollora, B. (1969) Long-term results of therapy in patients with chronic liver disease associated with hypergammaglobulinemia. American Journal of Medicine, 47, 765.

Read, A.E., Sherlock, S. \& Harrison, C.V. (1963) Active 'juvenile' cirrhosis considered as part of a systemic disease and the effect of corticosteroid therapy. Gut, 4, 378.

Slovis, T.E., Dubois, R.S., Rodgerson, D.O. \& SilverMAN, A. (1971) The varied manifestations of Wilson's disease. Journal of Pediatrics, 78, 578.

Starzl, T.E. (1969) Experience in Hepatic Transplantation, Ist edition. W. B. Saunders Co., Philadelphia.

Sternlieb, I. \& Scheinberg, I.H. (1972) Chronic hepatitis as a first manifestation of Wilson's disease. Annals of Internal Medicine, 76, 59.

Soloway, R.D., Baggenstoss, A.H., Schoenfield, L.J. \& SUMMERSKILL, W.H.J. (1971) Observer error and sampling variability in evaluation of hepatitis and cirrhosis by liver biopsy. American Journal of Digestive Disease, 16, 1082.

Soloway, R.D., Summerskill, W.H.J., Baggenstoss, A.H., Geall, M.G., Gitnick, G.L., Elveback, L.R. \& SchoenFIELD, L.J. (1972) Clinical, biochemical and histological remission of severe chronic active liver disease: A controlled study of treatment and early prognosis. Gastroenterology, 63, 820 . 


\section{Discussion}

Dialogue developed between Dr Javitt and Dr Dubois on the importance of the sex of the patient in the response to immuno-suppressants, particularly azathioprine. Dr Javitt took the view that there was no good evidence that males actually benefit from such therapy, for example, in the study from the Mayo clinic (Soloway et al., 1972), twelve of the thirteen patients who were treated with azathioprine alone were male and therefore one could almost have predicted that the outcome in these patients would not be satisfactory. In a study from the Royal Free (Cook et al., 1971), female patients predominated in the treated group. Dr Dubois agreed with these observations and said that the majority of their patients were in fact females, but he was not sure what the importance of this was since most of the patients were pre-pubertal. All eight who died were female. 